\title{
Study of the influence of the technological parameters on the weld quality using artificial neural networks
}

\author{
Daniel-Constantin Anghel ${ }^{1,{ }^{*}}$ and Alexandru Ene $^{2}$ \\ ${ }^{1}$ University of Pitesti, Department of Manufacturing and Industrial Management, Str. Tg. din Vale, \\ nr. 1, Pitesti, Romania Romania \\ ${ }^{2}$ University of Pitesti, Department of Electronics, Computers, Communications and Electrical \\ Engineering, Str. Tg. Din Vale, nr. 1, Pitesti, Romania
}

\begin{abstract}
This paper presents a study on the weld quality obtained by different values of the input parameters. The weld quality is characterized by two categories of parameters: geometrical parameters and mechanical parameters. They are dependent on the following process parameters: electric arc voltage, electric current intensity, welding speed, the feed wire velocity. Because the dependence between inputs and outputs is a nonlinear one was used an artificial feed forward neural network (ANN). The ANN was trained with the backpropagation algorithm, using as training patterns data measured from the mechanical process. This ANN can be used to estimate some parameters from future experiments of the mechanical process.
\end{abstract}

\section{Introduction}

Automatic welding is wide used to build bridges, train wagons, tanks, pipes and other products. Today, the clients are more and more exigent with the products. The manufacturers must give a distinct attention to the quality and to the characteristics of the product. For the pieces assembled by automatic welding process, the manufacturer must guarantee the weld quality, from the point of view geometrical and structural. The analyse of the welding parameters effect over the quality indexes shows that only a part of them has a significant effect to be considered and studied. The weld quality is characterized by the geometry $(B$ the maximum width of the welding cord [mm]; $b$ - weld reinforcement [mm], HV1OHAZ micro-hardness in the heat affected zone $[\mathrm{HV}]$ ) and they are dependent on the following process parameters: Iw - current intensity [A], Ua - electric arc voltage [V], Sa - wire feed speed $[\mathrm{cm} / \mathrm{min}]$, Ws - welding speed $[\mathrm{cm} / \mathrm{min}]$.

\section{Welding experimentation}

The material of the part is a steel for construction, S235JR (SR EN 10025-2:2004). The wire for welding was S 4T 0 AR SO (EN 756) with diameter of $4 \mathrm{~mm}$, and the fux was AS481.

\footnotetext{
*Corresponding author: daniel.anghel@upit.ro
} 
The model of the study must be chosen so that for a set of points in the experimental field we can know the value of the studied response. But, to make a thorough research we are restricted by the costs and time. For this reason, we cannot carry out experiments for the whole experimental field. The traditional methods provide a sequential workflow that makes the input factors in the studied system, automated welding flux welding, to be approached one after the other without prior planning in the resulting set of experiences. For this reason, it appears that the decision is prevented from occurring, and therefore it is necessary to continue experimentally. Thus, the answer is obtained step by step. The traditional method is known as the "tests with errors".

The limitations of traditional methods, "tests with errors", consist of:

- lead to incomplete conclusions from unknown causes;

- involves a long duration of experimentation;

- lead to the relative knowledge of the influence of the factors; values;

- does not allow predicted behavior of the studied system for untested parameter

For this study, for each output parameter a number of 40 experiments was chosen.

Table 1. The relationship between $\mathbf{B}$ and input parameters.

\begin{tabular}{|c|c|c|c|c|c|c|c|c|c|c|c|}
\hline \multirow{2}{*}{$\begin{array}{l}\text { Set } \\
\text { of } \\
\text { data }\end{array}$} & \multirow{2}{*}{$\mathrm{Ua}$} & \multirow{2}{*}{ Iw } & \multirow{2}{*}{ Ws } & \multirow{2}{*}{$\mathrm{Sa}$} & \multicolumn{5}{|c|}{$\mathrm{B}[\mathrm{mm}]$} & \multirow[b]{2}{*}{ Average } & \multirow{2}{*}{$\begin{array}{l}\text { Standard } \\
\text { deviation }\end{array}$} \\
\hline & & & & & 1 & 2 & 3 & 4 & 5 & & \\
\hline 1 & 28 & 500 & 112 & 120 & 16.5 & 15.5 & 15 & 15.2 & 14.2 & 15.3 & 0.83 \\
\hline 2 & 38 & 500 & 112 & 160 & 12.5 & 10 & 12.7 & 9.5 & 11.9 & 11.3 & 1.47 \\
\hline 3 & 28 & 650 & 112 & 160 & 14 & 14.3 & 14.8 & 14.6 & 14.2 & 14.4 & 0.31 \\
\hline 4 & 38 & 650 & 112 & 120 & 13.3 & 12.5 & 12.1 & 13.3 & 16.6 & 13.5 & 1.77 \\
\hline 5 & 28 & 500 & 168 & 160 & 9.8 & 9.5 & 10.8 & 10.4 & 10.1 & 10.1 & 0.50 \\
\hline 6 & 38 & 500 & 168 & 120 & 12.5 & 10.6 & 11.9 & 12 & 12.5 & 11.9 & 0.77 \\
\hline 7 & 28 & 650 & 168 & 120 & 11.9 & 11.5 & 11.1 & 11.8 & 11.6 & 11.6 & 0.31 \\
\hline 8 & 38 & 650 & 168 & 160 & 18.5 & 18.1 & 17.5 & 16.9 & 16.8 & 17.5 & 0.74 \\
\hline
\end{tabular}

Table 2. The relationship between $\mathbf{b}$ and input parameters.

\begin{tabular}{|c|c|c|c|c|r|r|r|r|r|c|c|}
\hline $\begin{array}{c}\text { Set } \\
\text { of } \\
\text { data }\end{array}$ & \multirow{2}{*}{ Ua } & \multirow{2}{*}{ Iw } & \multirow{2}{*}{ Ws } & Sa & & \multicolumn{7}{|c|}{1} & 2 & 3 & 4 & 5 & Average & $\begin{array}{c}\text { Standard } \\
\text { deviation }\end{array}$ \\
\hline 1 & 28 & 500 & 112 & 120 & 1.8 & 1.2 & 1.3 & 1.7 & 1.1 & 1.42 & 0.31 \\
\hline 2 & 38 & 500 & 112 & 160 & 2.2 & 1.4 & 2.2 & 3.2 & 1.3 & 2.06 & 0.76 \\
\hline 3 & 28 & 650 & 112 & 160 & 1.8 & 1.5 & 1.3 & 1.8 & 1 & 1.48 & 0.34 \\
\hline 4 & 38 & 650 & 112 & 120 & 3 & 2.5 & 1.5 & 3.4 & 1.5 & 2.38 & 0.86 \\
\hline 5 & 28 & 500 & 168 & 160 & 4.1 & 2.7 & 2.1 & 2.7 & 1.4 & 2.6 & 0.99 \\
\hline 6 & 38 & 500 & 168 & 120 & 2.8 & 3 & 2.7 & 2.9 & 2.7 & 2.82 & 0.13 \\
\hline 7 & 28 & 650 & 168 & 120 & 0.6 & 0.8 & 0.9 & 1.2 & 0.5 & 0.8 & 0.27 \\
\hline 8 & 38 & 650 & 168 & 160 & 2.5 & 1.9 & 2 & 2.6 & 1.9 & 2.18 & 0.34 \\
\hline
\end{tabular}

Table 3. The relationship between HV10HAZ and input parameters.

\begin{tabular}{|c|c|c|c|c|c|c|c|c|c|c|c|}
\hline \multirow{2}{*}{$\begin{array}{c}\text { Set } \\
\text { of } \\
\text { data }\end{array}$} & \multirow{2}{*}{ Ua } & Iw & Ws & Sa & \multicolumn{5}{|c|}{ HV10HAZ [HV] } & Average & $\begin{array}{c}\text { Standard } \\
\text { deviation }\end{array}$ \\
\hline 1 & 28 & 500 & 112 & 120 & 151 & 150 & 150 & 152 & 149 & 150.2 & 1.03 \\
\hline 2 & 38 & 500 & 112 & 160 & 166 & 153 & 157 & 170 & 179 & 165 & 10.36 \\
\hline
\end{tabular}




\begin{tabular}{|l|l|l|l|l|l|l|l|l|l|l|l|}
\hline 3 & 28 & 650 & 112 & 160 & 146 & 140 & 159 & 147 & 145 & 147.4 & 7.021 \\
\hline 4 & 38 & 650 & 112 & 120 & 149 & 143 & 152 & 164 & 151 & 151.8 & 7.66 \\
\hline 5 & 28 & 500 & 168 & 160 & 150 & 170 & 151 & 169 & 154 & 158.8 & 9.88 \\
\hline 6 & 38 & 500 & 168 & 120 & 151 & 149 & 153 & 155 & 150 & 151.6 & 2.41 \\
\hline 7 & 28 & 650 & 168 & 120 & 145 & 159 & 154 & 149 & 147 & 150.8 & 5.67 \\
\hline 8 & 38 & 650 & 168 & 160 & 148 & 143 & 145 & 134 & 139 & 141.8 & 5.44 \\
\hline
\end{tabular}

Some weld bead obtained in this experiment are showed in the Table 4.

Table 4. Some weld bead obtained in this experiment.

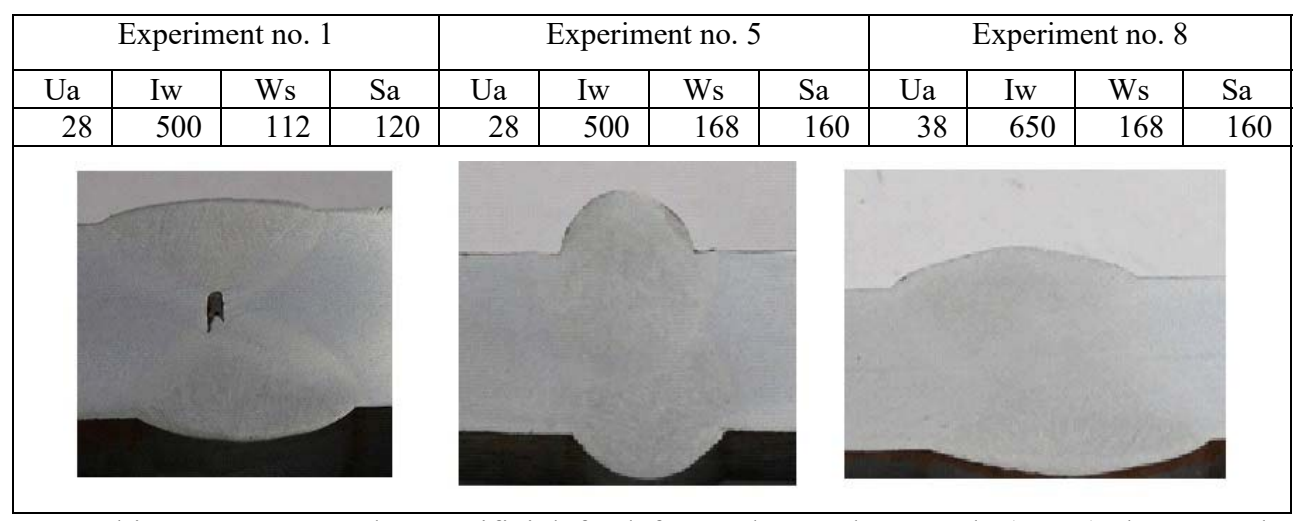

In this paper we used an artificial feed forward neural network (ANN), because the dependence between inputs and outputs is a nonlinear one.

\section{Feed forward neural networks}

An Artificial Neural Network (ANN) is composed of interconnected artificial neurons that mimic some properties of biological neurons [1]. There are many different types of neural networks, like: feed forward neural networks, Hopfield, Kohonen, etc. For this study, we used a feed forward neural network.

In feed forward neural networks, the artificial neurons are grouped in layers. Typically, there is an input layer, one or more hidden layers and an output layer. Each neuron from a layer is interconnected with all the neurons from the next layer, figure 3. To each connection is assigned a real number, called weight (positive or negative), which stands for the strength of the connection.

The size of the network is determined by the specific problem to be solved. So, if we have 3 input parameters, we used 3 input neurons. If we have 2 different output classes, we have 2 output neurons. The size of the hidden layer is experimentally chosen.

In order for neural networks to correctly classify an input pattern, they have to be trained with a set of examples. These set of training examples are taught by the network by applying the well-known backpropagation training algorithm. This algorithm calculates all the weights of the network.

In this application we simulated the feed forward neural network, using the MATLAB development environment.

MATLAB is a programming language and a development environment, which is based on matrix computations. For the development of neural network applications, it has the Neural Network toolbox. 


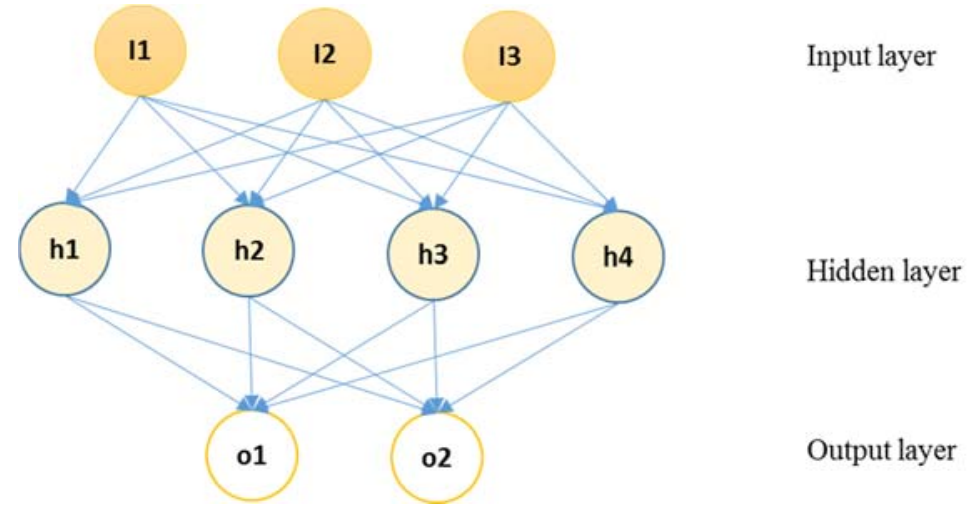

Fig. 1. A 3-4-2 feed forward neural network.

The parameters used to train the network are:

- Input parameters: Ua, Iw, Ws and Sa. Each of these parameters corresponds to an input neuron.

- Output parameter: B, b and HV10HAZ. Each of these parameters corresponds to an output neuron.

Were used 8 patterns [2].

Table 5. Dataset used to train, to test and to validate the network for the experiment.

\begin{tabular}{|c|c|c|c|c|c|c|c|}
\hline \multirow{2}{*}{ Set of data } & \multicolumn{4}{|c|}{ INPUTS } & \multicolumn{3}{c|}{ OUTPUTS } \\
\cline { 2 - 8 } & Ua & Iw & Ws & Sa & B [mm] & b [mm] & HV10HAZ [HV] \\
\hline 1 & 28 & 500 & 112 & 120 & 15.3 & 1.42 & 150.2 \\
\hline 2 & 38 & 500 & 112 & 160 & 11.3 & 2.06 & 165 \\
\hline 3 & 28 & 650 & 112 & 160 & 14.4 & 1.48 & 147.4 \\
\hline 4 & 38 & 650 & 112 & 120 & 13.5 & 2.38 & 151.8 \\
\hline 5 & 28 & 500 & 168 & 160 & 10.1 & 2.6 & 158.8 \\
\hline 6 & 38 & 500 & 168 & 120 & 11.9 & 2.82 & 151.6 \\
\hline 7 & 28 & 650 & 168 & 120 & 11.6 & 0.8 & 150.8 \\
\hline 8 & 38 & 650 & 168 & 160 & 17.5 & 2.18 & 141.8 \\
\hline
\end{tabular}

\section{The results}

In MATLAB, using Neural Network Toolbox, we have chosen the Fitting Tool from the Neural Network Start panel. This kind of tool is dedicated to build a neural network able to map between a data set of numeric inputs and a set of numeric targets. The Neural Network Fitting Tool help the specialists to select data, to create and to train a network, and to evaluate its performance using mean square error and regression analysis. The data was imported in this tool. For the input parameters, we have created a 4x8 matrix (Inputs) and for the output parameters, we have created a $3 \times 8$ matrix (Outputs). In order to training, to testing and to validate the network, we have divided the patterns into three categories: $70 \%$ of the patterns for training the network and for each one of others, testing and validation, $15 \%$ of the patterns.

The Neural Network Fitting Tool has the possibility to randomly distribute the patterns in all the three categories. The network was trained using "Bayesian-Regularization" back propagation algorithm [3], figure 2. This process is an iterative process, in this case, for training the network a number of 438 iterations was made. 


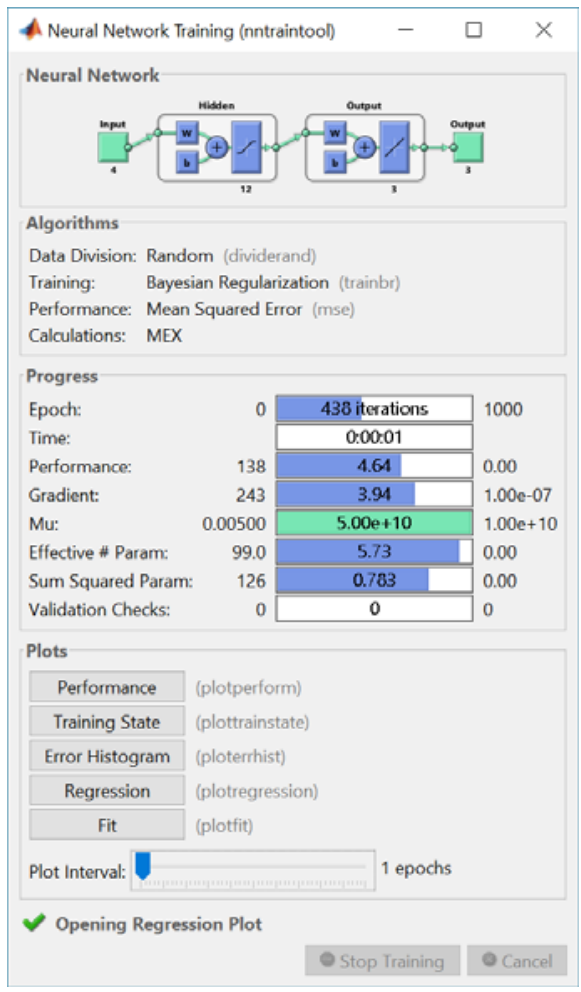

Fig. 2. The network training process whit MATLAB.

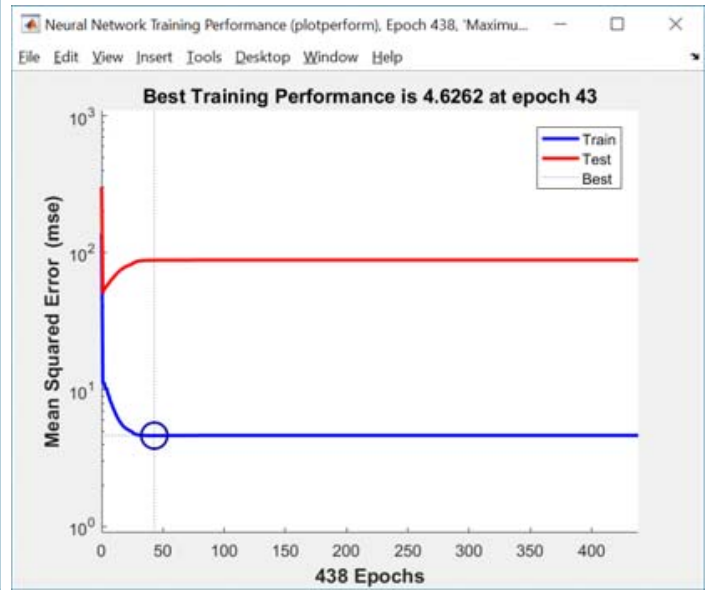

Fig. 3. The performance panel for the experiment.

Elle Edit Yiew Insert Iools Desktop Window Help
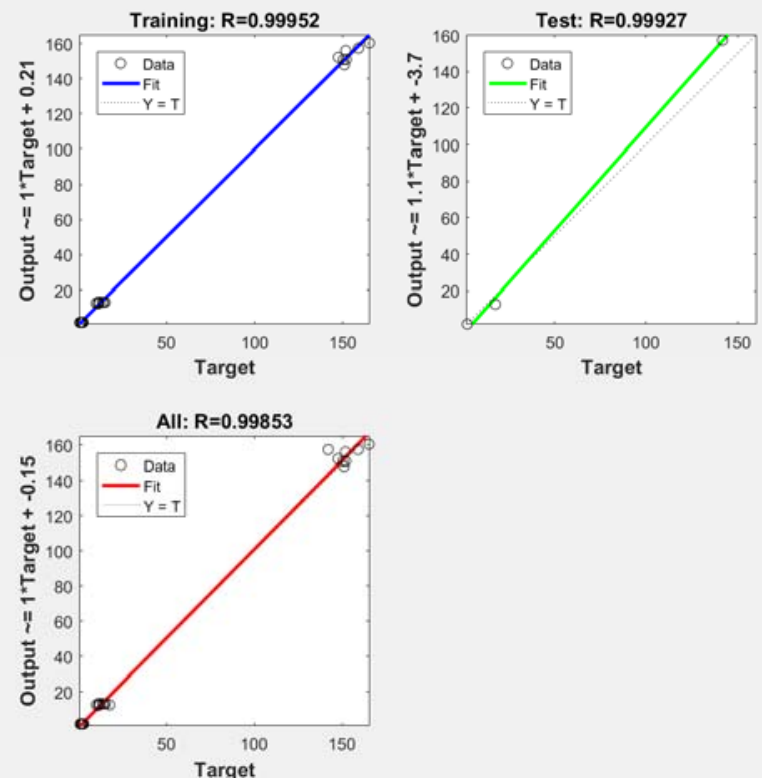

Fig. 4. The Regression panel for the experiment. 
When the training process is complete, on the Train Network panel the results are showed. In order to analyze the manner of progress of the training process, in the command line of the command window in MATLAB, we have used the function "plotperform".

This function plots the training, validation, and test performances given the training record returned by the function "train", figure 3 .

Figure 4 show the corresponding regressions diagrams for training and testing the network and a global regression for all stages.

\section{Conclusions}

In this paper, we used a feed forward neural network, to approximate the parameter of quality of the automatic welding process.

Using ANN allowed the study on a small number of samples than using a classical approach because ANN are able to learn from examples and are able to make connections.

This is an obvious advantage because it is economical and once a network is trained, it can be used in various simulations.

In the future we plan a more detailed approach, so the results to be richer and the external influencing factors to be identified and characterized more precisely.

\section{References}

1. A. Ene, C. Stirbu, ECAI 2016 (2016)

2. D-C. Anghel, N. Belu, N. Rachieru, AMR (2014)

3. A. Azadeh, M, Rouzbahman, M. Saberi \& I. M. Fam. JOLPPI, 24, 4 (2011) 